\title{
A review of lenalidomide in combination with dexamethasone for the treatment of multiple myeloma
}

\author{
Teru Hideshima' \\ Noopur Raje ${ }^{1,2}$ \\ Paul G Richardson' \\ Kenneth C Anderson' \\ 'Jerome Lipper Multiple Myeloma \\ Center, Dana-Farber Cancer Institute; \\ ${ }^{2}$ Massachusetts General Hospital \\ Cancer Center, Harvard Medical \\ School, Boston, MA, USA
}

\begin{abstract}
Lenalidomide (also known as Revlimid ${ }^{\circledR}$, CC-5013) is an immunomodulatory derivative of thalidomide and has more potent anti-tumor and anti-inflammatory effects than thalidomide. The molecular mechanisms of anti-tumor activity of lenalidomide have been extensively studied in multiple myeloma (MM) both preclinical models and in clinical trials. Lenalidomide: directly triggers growth arrest and/or apoptosis of drug resistant MM cells; inhibits binding of MM cells to bone marrow (BM) extracellular matrix proteins and stromal cells; modulates cytokine secretion and inhibits angiogenesis in the BM milieu; and augments host anti-tumor immunity. Lenalidomide achieved responses in patients with relapsed refractory MM. Moreover, lenalidomide with dexamethasone (Dex) demonstrates more potent anti-MM activities than Dex both in vitro and in randomized phase III clinical trials. Specifically, the combination improved overall and extent of response, as well as prolonged time to progression and overall survival, resulting in FDA approval of lenalidomide with Dex for therapy MM relapsing after prior therapy.
\end{abstract}

Keywords: lenalidomide, dexamethasone, multiple myeloma

\section{Introduction}

Multiple myeloma (MM) is a B cell malignancy characterized by excess monotypic plasma cells in the BM in association with monoclonal protein in serum and/or urine, decreased normal immunoglobulin (Ig) levels, and lytic bone disease. The 2006 estimate of multiple myeloma incidence in the United States is 16,570 cases, with an estimated number of 11,300 deaths. Conventional therapies with alkylating agents, anthracyclines, and corticosteroids can extend patient survival to a median of 3-4 years (Gregory et al 1992; Group 1998), and high dose therapy followed by autologous transplantation can modestly prolong median survival to 4-5 years (Fermand et al 1998; Lenhoff et al 2000). Attempts to improve autografting include repeated use of high dose therapies (Desikan et al 2000; Attal et al 2003), as well as immune strategies to treat minimal residual disease post-transplant (Massaia et al 1999) can improve outcome in some studies, few, if any, patients are cured. MM remains incurable due to the development of tumor cell resistance to all therapies, highlighting the urgent need for novel treatment strategies.

Thalidomide (Thal) has shown to be useful in various diseases including MM; however, it is a potent teratogen and causes side effects including peripheral neuropathy (Tseng et al 1996). Attempts were therefore made to develop Thal analogs which are more potent and have less adverse effects: lenalidomide $\left(\mathrm{C}_{13} \mathrm{H}_{13} \mathrm{~N}_{3} \mathrm{O}_{3}, \mathrm{MW}=259.26\right)$ is one such analog belonging to the class ofimmunomodulatory drugs (IMiDs) developed by the drug discovery program.
Correspondence: Teru Hideshima

Dana-Farber Cancer Institute, Mayer 549, 44 Binney Street, Boston, MA 02I I5, USA

Tel +| 6176322144

Fax + 6176322140

Email teru_hideshima@dfci.harvard.edu 


\section{Preclinical studies of lenalidomide Overview (Figure I)}

The interaction of multiple myeloma (MM) cells with bone marrow (BM) extracellular matrix (ECM) proteins and BM accessory cells, BM stromal cells (BMSCs), osteoblasts, osteoclasts, endothelial cells, as well as other factors in the BM milieu (ie, cytokines, angiogenesis) plays a crucial role in MM pathogenesis and drug resistance (Damiano et al 1999; Akiyama et al 2002; Hideshima and Anderson 2002; Hideshima et al 2003, 2004, 2006; Chauhan et al 2004). These accessory cells not only physically interact with MM cells, but also secrete growth and/or antiapoptotic factors such as interleukin (IL)-6, insulin-like growth factor (IGF)-1, vascular endothelial growth factor (VEGF), and tumor necrosis factor (TNF)- $\alpha$ (Akiyama et al 2002; Chauhan et al 1996, 2004, 2005; Catley et al 2004; Hideshima et al 2006). Delineation of the mechanisms of BM stromal cell (SC)-mediated MM cell proliferation, survival, drug resistance, and migration therefore provides the framework for identification and validation of novel therapeutic targets.
Within the BM microenvironment, several proliferative/ antiapoptotic signaling cascades are activated in MM cells: phosphatidylinositol-3 kinase (PI3K)/Akt (also known as protein kinase $\mathrm{B}$ ); I $\kappa \mathrm{B}$ kinase (IKK)/nuclear factor $\kappa-\mathrm{B}$ (NFKB); Ras/Raf/mitogen-activated protein kinase (MAPK) kinase (MEK)/extracellular signal-related kinase (ERK); and Janus kinase (JAK) 2/signal transducers and activators of transcription (STAT)-3 (Figure 1, Table 1). These signaling cascades mediate: cytoplasmic sequestration of many transcription factors; upregulation of cyclin D and anti-apoptotic Bcl-2 family members; as well as augmentation of telomerase activity (Hideshima et al 2001a; Akiyama et al 2002). Importantly, these molecular events are triggered by both MM cell adherence to BMSCs and by cytokines secreted from BMSCs (Dankbar et al 2000; Hideshima et al 2004; Mitsiades et al 2004). Cytokines secreted from MM cells and BMSCs and other cells may in turn further augment cytokine secretion.

Novel biologically based agents target not only the MM cell, but also MM cell-host interactions, cytokines, and their sequelae in the BM milieu. Thalidomide and

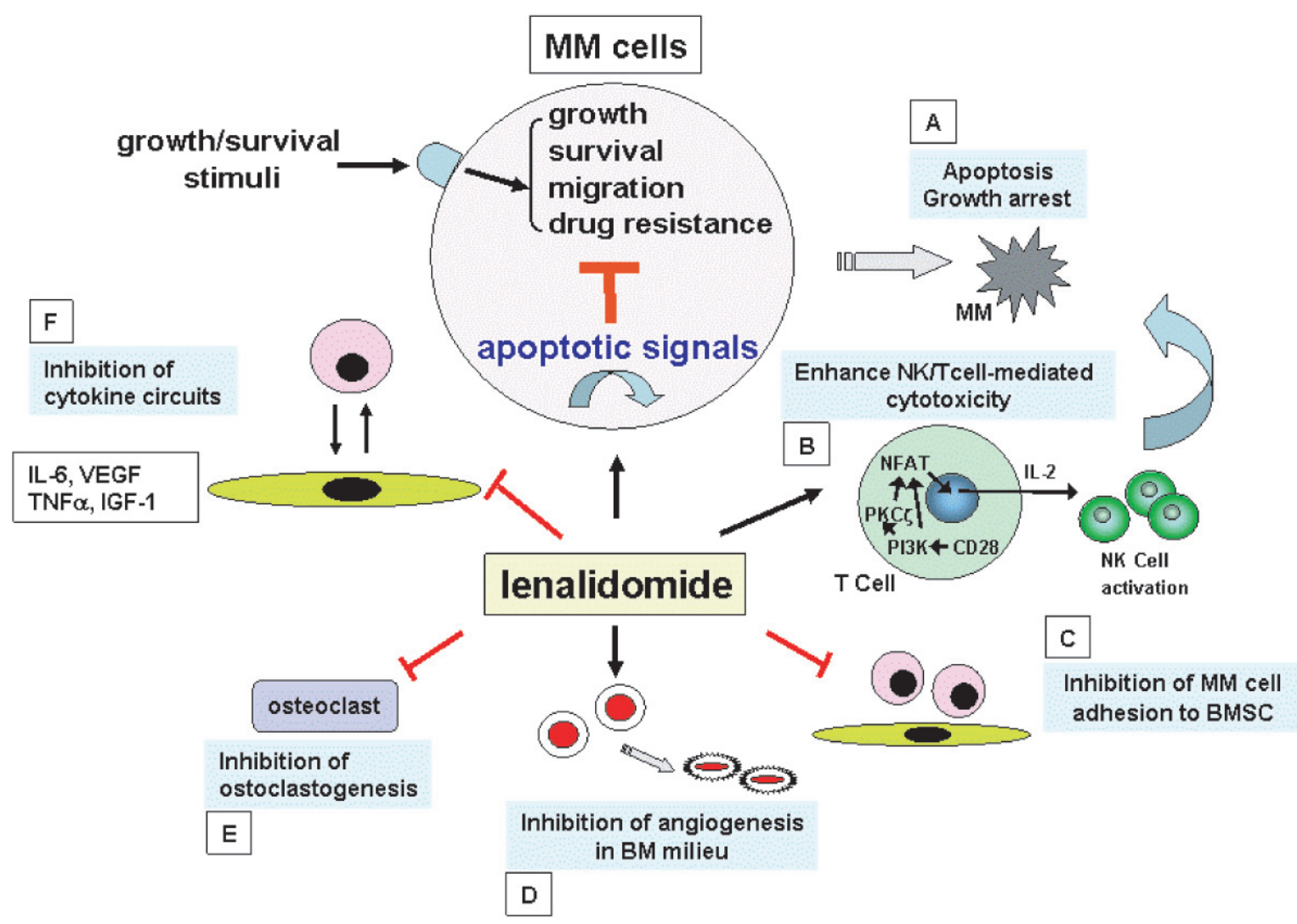

Figure I Potential mechanisms of action of anti-MM activity of lenalidomide. Lenalidomide: directly induces tumor cell apoptosis and/or growth arrest (A); enhances NK and/or NK cell activity via activation of CD28/NF-AT2 pathway (B); inhibits MM cell adhesion to host microenvironment (C); inhibits angiogenesis (D); inhibits osteoclastogenesis (E); as well as inhibits cytokine secretion $(\mathbf{F})$. 
its immunomodulatory derivative (IMiD) lenalidomide (Revlimid $^{\circledR}$; Celgene Corp., Summit, NJ, USA) are examples of such agents targeting the tumor cell in its BM milieu which can achieve responses even in refractory relapsed MM. Lenalidomide may inhibit MM cell growth by several different mechanisms (Figure 1). First, lenalidomide has a direct effect on MM cells to induce G1 growth arrest or apoptosis even of drug resistant cells (Hideshima et al 2000; Mitsiades et al 2002). Second, lenalidomide inhibits adhesion of MM cells to BMSCs, and thereby can overcome cell adhesion mediated drug resistance (CAM-DR); third, lenalidomide inhibits bioactivity and/or secretion in MM cells and/or BM stromal cells of cytokines [eg, interleukin (IL)-6, IL-1 $\beta$, IL-10, and tumor necrosis factor (TNF) $\alpha$ ] which augment MM cell growth, survival, drug resistance, migration, and expression of adhesion molecules. Importantly, lenalidomide is several thousand fold more potent than Thal at inhibiting TNF $\alpha /$ IL-1 $\beta$ secretion from mononuclear cells stimulated with lipopolysaccharide (LPS) in vitro (Corral et al 1999; Muller et al 1999). Fourth, vascular endothelial growth factor (VEGF) and basic fibroblast growth factor (bFGF) are secreted by MM cells and/or BMSCs, and lenalidomide may inhibit activity of VEGF, bFGF, and angiogenesis in MM. Lenalidomide also acts against MM through immunomodulatory effects such as augmentation of activity of cytotoxic T-cells and natural killer (NK) cells, associated with secretion of IL-2 and interferon- $\gamma$ (Davies et al 2001; LeBlanc et al 2004; Hayashi et al 2005).

Bone destruction is a hallmark of MM, with $70 \%-80 \%$ of patients manifesting bone involvement. Recently, Anderson et al demonstrated that an IMiD CC-4047 (Actimid ${ }^{\circledR}$; Celgene Corp., Summit, NJ, USA) inhibits osteoclastgenesis via downregulation of transcription factor PU.1 (Anderson et al 2006). Lenalidomide also has inhibitory effect on osteoclastogenesis (Terpos et al 2007).

Table I Selected ongoing clinical trials of lenalidomide based combination treatment in multiple myeloma

\begin{tabular}{lll}
\hline Agent & Phase & Patient \\
\hline Perifoine + Dex & I & Rel/ref \\
Hepatitis B vaccine & I & Rel/ref \\
Doxorubicin + Dex & I/II & Rel/ref \\
Bortezomib + Dex & I/II & Newly diagnosed \\
Bortezomib + Dex & II & Rel/ref \\
Bevacizumab + Dex & II & Rel/ref \\
Clarithromycin + Dex & II & Newly diagnosed \\
Dex & III & Newly diagnosed \\
Dex & III & Previously treated \\
Dex & IV & Previously treated \\
\hline
\end{tabular}

\section{Direct anti-tumor activities of lenalidomide}

Although the targets of whereby lelalidomide mediates antitumor activity of lenalidomide have not been fully delineated, several studies have examined the molecular mechanisms mediating sequelae of lenalidomide. Our previous studies demonstrated that lenalidomide induces $\mathrm{G} 0 / \mathrm{G} 1$ growth arrest associated with $\mathrm{p} 21^{\mathrm{Cip} 1}$ upregulation and/or apoptosis which is mediated via caspase-8 activation (Hideshima et al 2000; Mitsiades et al 2002). Lenalidomide inhibits LPS-mediated induction of Cox-2 and prostaglandin E2 (PGE2) production by a post-transcriptional mechanism in RAW 364.7 cells (Fujita et al 2001), suggesting that the anti-tumor activity induced by lenalidomide may also be due to inhibition of Cox-2 and PGE2. Lenalidomide inhibits nuclear factor (NF)- $\mathrm{KB}$ subunit activity in MM cell lines (Mitsiades et al 2002), which is consistent with reports that Thal inhibits DNA binding activity of the $\mathrm{p} 50 / \mathrm{p} 65 \mathrm{NF}-\mathrm{\kappa B}$ triggered by TNF $\alpha$ and IL- $1 \beta$ in Jurkat cell line (Keifer et al 2001) and in PBMCs (Rowland et al 2001). Since NF-KB plays an essential role in cell cycle regulation, cell survival, anti-apoptosis, and cytokine production in MM cells (Hideshima et al 2001b, 2002), inhibition of $\mathrm{NF}-\kappa \mathrm{B}$ activity by lenalidomide may also enhance or restore sensitivity to other chemotherapeutic agents. Specifically, we have demonstrated that MM cell adhesion-mediated upregulation of IL- 6 is mediated via NF- $\mathrm{\kappa B}$ activation (Chauhan et al 1996; Hideshima et al 2002). Recently, Stewart et al (2004) reported pharmacogenomic studies suggesting that hyperactivation of the Wnt signaling antagonist DKK-1 is associated with response to the immunomodulators Thal and lenalidomide. Furthermore, $\beta$-catenin expression is downregulated by lenalidomide in MM cell lines.

Lenalidomide in combination with Dex is one of the most promissing MM novel treatment options. It induces at least additive direct cytotoxicity in MM cells (Hideshima et al 2000), associated with activation of dual apoptotic signaling cascades: Dex induces caspase-9 (Chauhan et al 2001; Hideshima et al 2001a) and lenalidomide triggers caspase- 8 activation (Mitsiades et al 2002) (Figure 2). Most recently, enhanced anti-MM activity of rapamycin, a specific mTOR inhibitor, in combination with lenalidomide has been reported (Raje et al 2004). In this study, the combination of rapamycin plus lenalidomide overcomes drug resistance in MM cell lines resistant to conventional chemotherapy. Interestingly, differential signaling cascades, including the ERK and PI3$\mathrm{K} /$ Akt pathways, are targeted by these drugs individually and in combination, suggesting the molecular mechanism by which they inhibits MM growth and survival. 


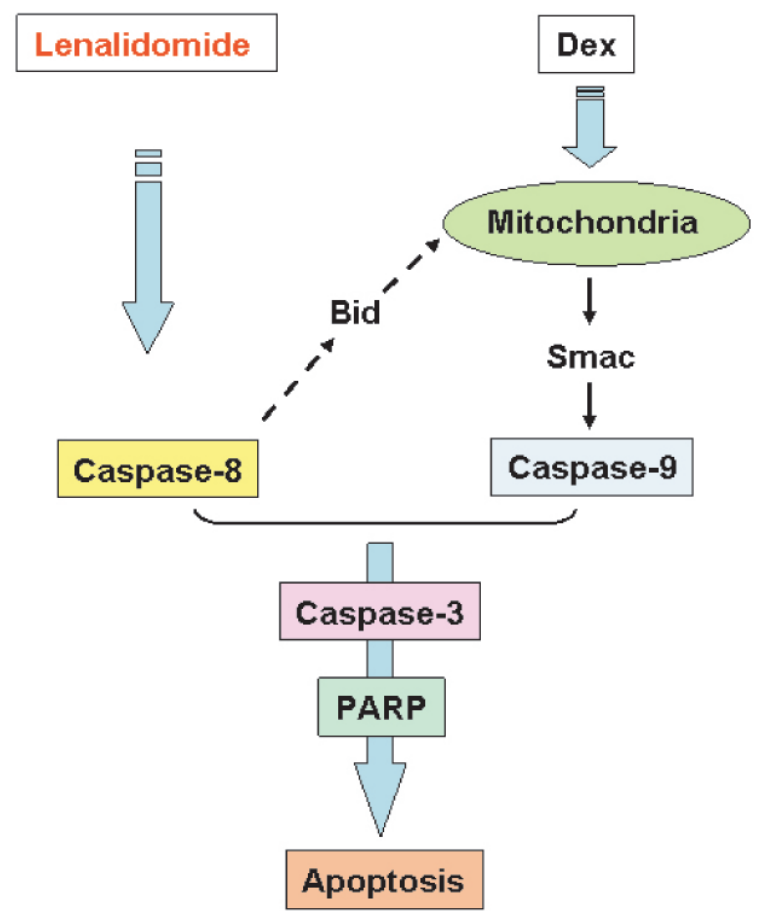

Figure 2 Potential mechanisms of synergistic cytotoxicity by lenalidomide plus Dex treatment in MM cells. Lenalidomide triggers caspase-8 dependent apoptosis, whereas Dex induces caspase- 9 dependent apoptosis. The combination therefore triggers dual apoptotic signaling cascades.

\section{Anti-angiogenic activity}

Previous studies have shown that oral administration of lenalidomide attenuates growth factor-induced angiogenesis in vivo. This effect is correlated with the inhibitory effect of lenalidomide on growth factor-induced Akt phosphorylation, thereby providing a potential mechanism for its antimigratory and subsequent anti-angiogenic effects (Dredge et al 2005). In MM, an anti-angiogenic effect of Thal in vitro has been demonstrated (D'Amato et al 1004; Singhal et al 1999; Lentzsch et al 2002; Fujita et al 2004); however, to date no strong evidence of an anti-angiogenic effect of lenalidomide in vivo has been demonstrated. Moreover, Singhal et al (1999) reported no correlation of BM angiogenesis with response to Thal in patients with relapsed refractory MM, suggesting that lenalidomide may mediate its anti-MM activity via mechanisms other than anti-angiogenesis.

\section{Immunomodulatory activities}

A unique feature of the anti-tumor effect of Thal and lenalidomide is their ability to modulate and potentiate host immune responses against MM. Several studies have demonstrated the effects of lenalidomide on peripheral blood lymphocytes (Davies et al 2001; Haslett et al 2003; LeBlanc et al 2004; Hayashi et al 2005). Co-culture of naive splenocytes with
anti-CD3 monoclonal antibody and IMiD1 (Actimid ${ }^{\circledR}$ ) directly costimulates $\mathrm{T}$ cells and increases Th-1-type cytokines. Most excitingly, IMiDs augment CTL and NK cell activity against MM cell lines and autologous MM cells, associated with increased IL-2 levels in serum (Davies et al 2001). Although Thal/IMiDs induce IL-2 secretion from T cells (Corral et al 1999; Shannon et al 2000), the mechanisms whereby these compounds induce IL-2 production from $\mathrm{T}$ cells has not totally been defined. Importantly, our recent studies demonstrated that lenalidomide significantly costimulates proliferation of CD3 $+\mathrm{T}$ cells induced by $\mathrm{CD} 3$ ligation, immature dendritic cells (DCs; SI, 2.1), or mature DCs (SI, 2.6). T-cell proliferation triggered by DCs is abrogated by cytotoxic $\mathrm{T}$ lymphocyte antigen 4-immunoglobulin (CTLA-4-Ig). Lenalidomide also overcomes the inhibitory effects of CTLA-4-Ig on Epstein-Barr virus and influenzaspecific CD4 and CD8 T-cell responses, as measured by cytokine capture and enzyme-linked immunosorbent spot (ELISPOT) assays. Importantly, lenalidomide triggers tyrosine phosphorylation of CD28 on T cells, followed by activation of NF- $\kappa B$ (LeBlanc et al 2004). Furthermore, we have demonstrated that IMiDs facilitate the nuclear translocation of nuclear factor of activated T cells (NF-AT)-2 and activator protein-1 via activation of PI3-K/Akt signaling, with resultant IL-2 secretion. IMiDs enhance both NK cell cytotoxicity and ADCC induced by triggering IL-2 production from T cells (Hayashi et al 2005). These studies therefore define the molecular mechanisms whereby lenalidomide triggers NK cell-mediated cytotoxicity against MM cells, further supporting their therapeutic use in MM. More recently, we have shown that lenalidomide enhances ADCC induced by SGN-40, a humanized IgG1 anti-CD40 monoclonal antibody (Tai et al 2005).

\section{Clinical studies of lenalidomide Pharmacokinetics}

Pharmacokinetics (PK) of lenalidomide in MM patients has been reported by Wu and Scheffler (2004) at American Society of Clinical Oncology in 2004. In this single-center, open-label, non-randomized, phase I dose escalation study in relapsed and refractory MM, the doses of lenalidomide used were $5,10,25$ or $50 \mathrm{mg} /$ day orally for 28 days. Blood samples were collected before and at $15 \mathrm{~min}, 30 \mathrm{~min}, 45 \mathrm{~min}$, 1 h, 1.5 h, 2 h, 2.5 h, 3 h, 4 h, 6 h, 8 h, 10 h, 12 h, 18 h, 24 h, $48 \mathrm{~h}$, and $72 \mathrm{~h}$ after administration on both days 1 and 28 . No lenalidomide dose-limiting toxicity was observed at any dose level within the first 28 days. Absorption of lenalidomide was rapid on both day 1 and 28 , with $\mathrm{t}_{\text {max }}$ ranging from 0.7 to $2.0 \mathrm{~h}$ 
at all dose levels. Plasma levels of lenalidomide declined in a monophasic manner, with elimination half-life ranging from 2.8 to $6.1 \mathrm{~h}$ on both days 1 and 28 at all four doses. No plasma accumulation was observed upon multiple dosing. Importantly, daily oral doses of lenalidomide up to $50 \mathrm{mg}$ produced no dose-limiting toxicity within the first 28 days.

The other PK study has been reported by Richardson et al (2006). In this study, plasma concentration of lenalidomide was determined in 39 patients during the first and second cycles in both $15 \mathrm{mg}$ and $30 \mathrm{mg}$ dose groups, and when Dex was added due to progressive disease (PD) or stable disease (SD) on lenalidomide alone. The mean minimum $\left(\mathrm{C}_{\min }\right)$ plasma lenalidomide concentrations on days $1,2,3,4$, and 21 during the first and second 21-day cycles of lenalidomide alone and with the addition of Dex are shown for the $30 \mathrm{mg}$ once-daily and $15 \mathrm{mg}$ twice-daily cohorts. The average $\mathrm{C}_{\text {min }}$ plasma levels were less in the twice-daily compared with daily dosing regimens. No obvious effect on lenalidomide plasma concentrations was seen with addition of Dex in either once- or twice-daily treatment.

\section{Clinical trials of lenalidomide}

Only a limited number of reports are available for clinical studies of lenalidomide (Bartlett et al 2004). A phase I clinical study of lenalidomide was completed at Dana-Farber Cancer Institute (Richardson et al 2002a). In this study, dose-escalation ( $5 \mathrm{mg} /$ day, $10 \mathrm{mg} /$ day, $25 \mathrm{mg} /$ day, and $50 \mathrm{mg}$ /day) of lenalidomide was evaluated in 27 patients (median age 57 years; range, 40-71 years) with relapsed and refractory relapsed MM (Richardson et al 2002b). These patients received a median of 3 (range, 2-6) prior regimens, including autologous stem cell transplantation and Thal in 15 and 16 patients, respectively. In 24 evaluable patients, no dose-limiting toxicity (DLT) was observed in patients treated at any dose level within the first 28 days; however, grade 3 myelosuppression developed after day 28 in all 13 patients treated with $50 \mathrm{mg} /$ day lenalidomide. Dose reduction to $25 \mathrm{mg}$ /day was well tolerated in 12 patients and therefore considered to be the maximal tolerated dose (MTD). Most importantly, no significant somnolence, constipation, or neuropathy, the most common toxicities of Thal, have been seen in any cohort. Best responses of at least $25 \%$ reduction in paraprotein occurred in 17 of $24(71 \%)$ patients $(90 \%$ confidence interval [CI], 52\%-85\%), including $11(46 \%)$ patients who had received prior Thal; stable disease (less than $25 \%$ reduction in paraprotein) was observed in an additional $2(8 \%)$ patients. This study therefore demonstrates that lenalidomide can overcome conventional drug resistance, even resistance to Thal. Given that lenalidomide is an oral agent, it is currently being evaluated in a randomized trial post autografting in an attempt to prolong progression free and overall survival.

A multicenter, open-label, randomized phase II study to evaluate 2 dose regimens of lenalidomide for relapsed, refractory MM has been performed. In this study, 70 patients were randomized to receive either $30 \mathrm{mg}$ once-daily or $15 \mathrm{mg}$ twice-daily oral lenalidomide for 21 days of every 28-day cycle. An additional 32 patients received $30 \mathrm{mg}$ once daily. Patients with progressive or stable disease after 2 cycles received additional Dex. Responses were evaluated according to European Group for Blood and Marrow Transplantation (EBMT) criteria. Overall response rate $(\mathrm{CR}+\mathrm{PR}+\mathrm{MR})$ to lenalidomide alone was $25 \% ; 24 \%$ for $30 \mathrm{mg}$ once-daily and $29 \%$ for $15 \mathrm{mg}$ twice-daily cohort. Median overall survival in 30-mg once-daily and $15 \mathrm{mg}$ twice-daily groups was 28 and 27 months, respectively. However, median progression-free survival was 7.7 months on $30 \mathrm{mg}$ once-daily versus 3.9 months on $15 \mathrm{mg}$ twice-daily lenalidomide. Dex was added in 68 patients and $29 \%$ responded. Importantly, time to first occurrence of clinically significant grade $3 / 4$ myelosuppression was shorter in the $15 \mathrm{mg}$ twice-daily group (1.8 months) than $30 \mathrm{mg}$ once-daily (5.5 months, $\mathrm{p}=0.05$ ) group. Moreover, analysis of the first 70 patients showed increased grade 3/4 myelosuppression in patients receiving $15 \mathrm{mg}$ twice-daily ( $41 \%$ vs $13 \%, p=0.03$ ). This study indicate that lenalidomide is active and well tolerated in relapsed, refractory myeloma, with the 30-mg once-daily regimen providing the basis for future studies as monotherapy and with Dex (Richardson et al 2006).

\section{Clinical studies of lenalidomide in combination with Dex}

As described above, preclinical studies have demonstrated the efficacy of combination treatment of lenalidomide with Dex in MM and several clinical trials of this combination treatment have been completed.

In two double blind, multicenter, international phase III clinical trials (MM-009, North American, 353 patients; MM-010, Europe, Australia, and Israel, 351 patients), patients with relapsed or refractory MM not resistant to Dex were treated with Dex $40 \mathrm{mg}$ daily on days 1-4, 9-12, and 17-20 every 28 days and were randomized to receive either lenalidomide $25 \mathrm{mg}$ daily orally on days 1-21 every 28 days or placebo. At a median follow-up from randomization of 17.1 months (MM-009) and 16.5 months (MM-010), both studies show significant improvement with lenalidomide 
plus Dex compared to Dex in overall response (OR) (MM-009: $1 \%$ vs $20.5 \%, \mathrm{p}<0.001$; MM-010: 59.1\% vs $24 \%, \mathrm{p}<0.001$, respectively), time to progression (TTP) (MM-009: 11.1 months vs 4.7 months, $\mathrm{p}<0.001$; MM-010: 11.3 months vs 4.7 months, $\mathrm{p}<0.001$, respectively), and overall survival (OS) (MM-009: 29.6 months vs 20.5 months, $\mathrm{p}<0.001$; MM-010: not estimable vs 20.6 months, $\mathrm{p}<0.001$, respectively). In a subgroup analysis on patients with impaired creatinine clearance, no significant difference in response rate, TTP, or OS was observed in patients with creatinine clearance above or below $50 \mathrm{~mL} /$ min who were treated with lenalidomide plus Dex; however, for 16 patients with creatinine clearance $<30 \mathrm{~mL} / \mathrm{min}$, median TTP and OS was shorter than for those with creatinine clearance $>30 \mathrm{~mL} / \mathrm{min}$, but still significantly longer than for patients treated with Dex (Weber et al 2006).

Treatment with lenalidomide plus Dex in newly diagnosed MM patients has also reported by Rajkumar et al (2005). In this study, lenalidomide was given orally $25 \mathrm{mg}$ daily on days 1-21 of a 28 -day cycle. Dex was given orally $40 \mathrm{mg}$ daily on days 1-4, 9-12, and 17-20 of each cycle. Thirty-one of 34 patients achieved an objective response, including $2(6 \%)$ achieving complete response (CR) and 11 (32\%) meeting criteria for both very good partial response and near complete response, resulting in an overall objective response rate of $91 \%$. This study indicated that lenalidomide plus Dex is a highly active regimen with manageable side effects in the treatment of newly diagnosed MM.

A number of studies demonstrated that MM is characterized by cytogenetic abnormalities causing dysregulation of the genes at the breakpoints, and by point mutations (Kuehl et al 2002; Fonseca et al 2004; Carrasco et al 2006). Specifically, chromosome 13 deletions are present in over $50 \%$ of MM patients and considered to be associated with poor prognosis. In addition, $\mathrm{t}(4 ; 14)$ in $\mathrm{MM}$ also predicts poor response to conventional and high dose treatment and shortened survival. A recent study has shown that lenalidomide overcomes the poor prognosis conferred by chromosome 13 deletion and $\mathrm{t}(4 ; 14)$ in MM patients, evidenced by event free survival and response rate (Bahlis et al 2006).

Recently, a phase I/II 3 combination treatment of lenalidomide, Dex and adriamycin (RAD therapy) for relapsed MM patient has been reported. In this study, 31 patients were evaluated for response and toxicity: 26 patients achieved reduction of paraprotein levels of at least $50 \%$ for a response rate of $84 \%$, including one confirmed CR and 14 PRs according to the EBMT criteria. Importantly, 8 of 10 patients who displayed del (13) on cytogenetic analysis responded, including 6 confirmed PRs. One patient each experienced acute renal failure due to emesis and hypovolemia, pneumocystis pneumonitis, and catheter related infection. Somnolence, constipation, thromboembolism, or neuropathy was not observed. This study showed that RAD induces substantial responses with an acceptable toxicity profile, and thus significantly contributes to the therapeutic armamentarium even in heavily pretreated MM patients (Knop et al 2006). Most recently, a phase I study of lenalidomide and dexamethasone in combination with Akt inhibitor perifosine for patients with relapsed or refractory MM, and a phase I/II study of lenalidomide, dexamethasone and bortezomib combination therapy for newly diagnosed MM patients are ongoing.

The common side effects of lenalidomide treatment in phase 2 clinical trials of relapsed refractory MM are summarized in Table 1. The most common toxicities associated with Thal (eg, constipation, neuropathy, tremors) were not observed. Toxicities associated with lenalidomide were primarily hematologic and reversible. The most common grade 3 or higher adverse events during lenalidomide therapy were neutropenia and thrombocytopenia. Grade 4 neutropenia occurred in 2 of $34(5.8 \%)$ patients treated at $15 \mathrm{mg}$ twice daily vs 4 of $68(5.9 \%)$ patients treated with $30 \mathrm{mg}$ daily. Grade 4 thrombocytopenia occurred in $2(5.8 \%)$ of 34 patients on $15 \mathrm{mg}$ twice daily vs 2 of 68 patients (2.9\%) treated with $30 \mathrm{mg}$ daily. Deep vein thrombosis (DVT) was reported in 1 patient on the $30 \mathrm{mg}$ daily and 2 patients (5.8\%) on the $15 \mathrm{mg}$ twice-daily treated regimen. Sedation or neurologic toxicities were not observed in most of these studies (Richardson et al 2006). The differences in the side effect profile between Thal and lenalidomide may reflect distinct patterns of antiangiogenic, cytokine-related, microenvironmental, and immunomodulatory activity, rather than distinct separate mechanisms of action.

In phase III trials of lenalidomide plus Dex for newly diagnosed MM patients, $47 \%$ of patients experienced grade III or higher nonhematologic toxicity. The most common adverse effects were fatigue (15\%), muscle weakness (6\%), anxiety (6\%), pneumonitis (6\%), and rash (6\%) (Rajkumar et al 2006a). Recently, a randomized phase III trial of lenalidomide plus high-dose Dex versus lenalidomide plus low-dose Dex in newly diagnosed MM has also reported by Rajkumar et al (2006b). In this study, patients in both arms received lenalidomide $25 \mathrm{mg}$ /day orally on days $1-21$ every 28 days. In addition, patients in the high-dose Dex arm received Dex $40 \mathrm{mg}$ on days 1-4, 9-12, and 17-20 orally every 28 days, while patients in the low-dose Dex 
arm received Dex $40 \mathrm{mg}$ on days $1,8,15$, and 22 orally every 28 days. Although response rate (RR) has not yet been reported, toxicity rates are higher in the high-dose Dex arm than low-dose Dex arm. For example, Grade 3 and above toxicities in cardiac ischemia $(2.7 \%$ vs $0.5 \%)$, hypercalcemia (5.8\% vs $1.8 \%$ ), infection (18.8\% vs $9 \%$ ), thromboembolism (18.4\% vs 5.4\%), and non-hematologic toxicities (22\% vs $12.6 \%$ ) are higher in high-dose Dex arm than low-dose Dex arm. If RR is similar in both arms, dosage of Dex can be reduced to $25 \mathrm{mg}$.

\section{Conclusion}

Lenalidomide plus Dex treatment is highly effective in both preclinical and clinical studies. It is one of the promising treatment options against both relapsed/refractory and newly diagnosed MM patients. Adverse effects of this combination can be markedly reduced by lowering the Dex dosage.

\section{Future directions}

Lenalidomide plus Dex treatment can be further combined with other novel or conventional agents to improve patient outcome in MM. Indeed, potent Akt inhibitor Perifosine, proteasome inhibitor bortezomib (Velcade ${ }^{\circledR}$; Millennium Pharmaceuticals Inc.), and anti-angiogenic agent bevacizumab (Avastin ${ }^{\circledR}$; Genentech) are already under evaluation in combination clinical trials.

\section{Acknowledgments}

Supported by National Institutes of Health Grant PO-1 78378 and RO-1 CA 50947; the Doris Duke Distinguished Clinical Research Scientist Award (KCA); the Multiple Myeloma Research Foundation (TH, NR, KCA); and the Myeloma Research Fund (KCA).

\section{References}

Akiyama M, Hideshima T, Hayashi T, et al. 2002. Cytokines modulate telomerase activity in a human multiple myeloma cell line. Cancer Res, 62:3876-82.

Anderson G, Gries M, Kurihara N, et al. 2006. Thalidomide derivative CC-4047 inhibits osteoclast formation by down-regulation of PU.1. Blood, 107:3098-105.

Attal M, Harousseau JL, Facon T, et al. 2003. Single versus double autologous stem-cell transplantation for multiple myeloma. $N \mathrm{Engl} \mathrm{J} \mathrm{Med}$, 349:2495-502.

Bahlis NJ, Mansoor A, Lategan JC, et al. 2006. Lenalidomide overcomes poor prognosis conferred by deletion of chromosome 13 and $\mathrm{t}(4 ; 14)$ in multiple myeloma: MM016 Trial. Blood, 108:1016a.

Bartlett JB, Dredge K, Dalgleish AG. 2004. The evolution of thalidomide and its IMiD derivatives as anticancer agents. Nat Rev Cancer, 4:314-22.

Carrasco DR, Tonon G, Huang Y, et al. 2006. High-resolution genomic profiles define distinct clinico-pathogenetic subgroups of multiple myeloma patients. Cancer Cell, 9:313-25.
Catley L, Tai YT, Shringarpure R, et al. 2004. Proteasomal degradation of topoisomerase I is preceded by c-Jun NH2-terminal kinase activation, Fas up-regulation, and poly(ADP-ribose) polymerase cleavage in SN38-mediated cytotoxicity against multiple myeloma. Cancer Res, 64:8746-53.

Chauhan D, Catley L, Li G, et al. 2005. A novel orally active proteasome inhibitor induces apoptosis in multiple myeloma cells with mechanisms distinct from Bortezomib. Cancer Cell, 8:407-19.

Chauhan D, Hideshima T, Rosen S, et al. 2001. Apaf-1/cytochrome c independent and Smac dependent induction of apoptosis in multiple myeloma cells. J Biol Chem, 276:24453-6.

Chauhan D, Li G, Hideshima T, et al. 2004. Blockade of ubiquitinconjugating enzyme CDC34 enhances anti-myeloma activity of Bortezomib/Proteasome inhibitor PS-341. Oncogene, 23:3597-602.

Chauhan D, Uchiyama H, Akbarali Y, et al. 1996. Multiple myeloma cell adhesion-induced interleukin- 6 expression in bone marrow stromal cells involves activation of NF-kB. Blood, 87:1104-12.

Corral LG, Haslett PAJ, Muller GW, et al. 1999. Differential cytokine modulation and $\mathrm{T}$ cell activation by two distinct classes of thalidomide analogues that are potent inhibitors of TNF- $\alpha$. J Immunol, 163:380-6.

D'Amato RJ, Loughman MS, Flynn E, et al. 1994. Thalidomide is an inhibitor of angiogenesis. Proc Natl Acad Sci USA, 91:4082-5.

Damiano JS, Cress AE, Hazlehurst LA, et al. 1999. Cell adhesion mediated drug resistance (CAM-DR): Role of integrins and resistance to apoptosis in human myeloma cell lines. Blood, 93:1658-67.

Dankbar B, Padro T, Leo R, et al. 2000. Vascular endothelial growth factor and interleukin-6 in paracrine tumor-stromal cell interactions in multiple myeloma. Blood, 95:2630-6.

Davies FE, Raje N, Hideshima T, et al. 2001. Thalidomide and immunomodulatory derivatives augment natural killer cell cytotoxicity in multiple myeloma. Blood, 98:210-16.

Desikan R, Barlogie B, Sawyer J, et al. 2000. Results of high-dose therapy for 1000 patients with multiple myeloma: durable complete remissions and superior survival in the absence of chromosome 13 abnormalities. Blood, 95:4008-10.

Dredge K, Horsfall R, Robinson SP, et al. 2005. Orally administered lenalidomide (CC-5013) is anti-angiogenic in vivo and inhibits endothelial cell migration and Akt phosphorylation in vitro. Microvasc Res, 69:56-63.

Fermand J-P, Ravaud P, Chevret S, et al. 1998. High-dose therapy and autologous peripheral blood stem cell transplantation in multiple myeloma: Up-front or rescue treatment? Results of a multicenter sequential randomized clinical trial. Blood, 92:3131-6.

Fonseca R, Barlogie B, Bataille R, et al. 2004. Genetics and cytogenetics of multiple myeloma: a workshop report. Cancer Res, 64:1546-58.

Fujita J, Mestre JR, Zeldis JB, et al. 2001. Thalidomide and its analogues inhibit lipopolysaccharide-mediated Iinduction of cyclooxygenase-2. Clin Cancer Res, 7:3349-55.

Fujita K, Asami Y, Tanaka K, et al. 2004. Anti-angiogenic effects of thalidomide: expression of apoptosis-inducible active-caspase-3 in a three-dimensional collagen gel culture of aorta. Histochem Cell Biol, 122:27-33.

Gregory WM, Richards MA, Malpas JS. 1992. Combination chemotherapy versus melphalan and prednisolone in the treatment of multiple myeloma: an overview of published trials. J Clin Oncol, 10:334-42.

Group MTsC. 1998. Combination chemotherapy versus melphalan plus prednisone as treatment for multiple myeloma: an overview of 6,633 patients from 27 randomized trials. J Clin Oncol, 16:3832-42.

Haslett PA, Hanekom WA, Muller G, et al. 2003. Thalidomide and a thalidomide analogue drug costimulate virus-specific CD8+ T cells in vitro. $J$ Infect Dis, 187:946-55.

Hayashi T, Hideshima T, Akiyama M, et al. 2005. Molecular mechanisms whereby immunomodulatory drugs activate natural killer cells: clinical application. Br J Haematol, 128:192-203.

Hideshima T, Anderson KC. 2002. Molecular mechanisms of novel therapeutic approaches for multiple myeloma. Nat Rev Cancer, 2:927-37. 
Hideshima T, Bergsagel PL, Kuehl WM, et al. 2004. Advances in biology of multiple myeloma: clinical applications. Blood, 104:607-18.

Hideshima T, Catley L, Yasui H, et al. 2006. Perifosine, an oral bioactive novel alkylphospholipid, inhibits Akt and induces in vitro and in vivo cytotoxicity in human multiple myeloma cells. Blood, 107:4053-62.

Hideshima T, Chauhan D, Richardson P, et al. 2002. NF- $\kappa B$ as a therapeutic target in multiple myeloma. $J$ Biol Chem, 277:16639-47.

Hideshima T, Chauhan D, Shima Y, et al. 2000. Thalidomide and its analogues overcome drug resistance of human multiple myeloma cells to conventional therapy. Blood, 96:2943-50.

Hideshima T, Nakamura N, Chauhan D, et al. 2001. Biologic sequelae of interleukin-6 induced PI3-K/Akt signaling in multiple myeloma. Oncogene, 20:5991-6000.

Hideshima T, Richardson P, Anderson KC. 2003. Novel therapeutic approaches for multiple myeloma. Immunol Rev, 194:164-76.

Hideshima T, Richardson P, Chauhan D, et al. 2001. The proteasome inhibitor PS-341 inhibits growth, induces apoptosis, and overcomes drug resistance in human multiple myeloma cells. Cancer Res, 61:3071-6.

Keifer JA, Guttridge DC, Ashburner BP, et al. 2001. Inhibition of NF- $\mathrm{kB}$ activity by thalidomide through suppression of IkappaB kinase activity. J Biol Chem, 276:22382-7.

Knop S, Gerecke C, Topp MS, et al. 2006. Lenalidomide (Revlimid), adriamycin and dexamethasone chemotherapy (RAD) is safe and effective in treatment of relapsed multiple myeloma -first results of a German multicenter phase I/II trial. Blood, 108:125a.

Kuehl WM, Bergsagel PL. 2002. Multiple myeloma: evolving genetic events and host interactions. Nat Rev Cancer, 2:175-87.

LeBlanc R, Hideshima T, Catley LP, et al. 2004. Immunomodulatory drug costimulates T cells via the B7-CD28 pathway. Blood, 103:1787-90.

Lenhoff S, Hjorth M, Holmberg E, et al. 2000. Impact on survival of highdose therapy with autologous stem cell support in patients younger than 60 years with newly diagnosed multiple myeloma: a population-based study. Blood, 95:7-11.

Lentzsch S, Rogers MS, LeBlanc R, et al. 2002. S-3-Amino-phthalimidoglutarimide inhibits angiogenesis and growth of B-cell neoplasias in mice. Cancer Res, 62:2300-5.

Massaia M, Borrione P, Battaglio S, et al. 1999. Idiotype vaccination in human myeloma: generation of tumor-specific immune responses after high-dose chemotherapy. Blood, 94:673-83.

Mitsiades CS, Mitsiades N, Munshi NC, et al. 2004. Focus on multiple myeloma. Cancer Cell, 6:439-44.

Mitsiades N, Mitsiades CS, Poulaki V, et al. 2002. Apoptotic signaling induced by immunomodulatory thalidomide analogs in human multiple myeloma cells: therapeutic implications. Blood, 99:4525-30.

Muller GW, Chen R, Huang SY, et al. 1999. Amino-substituted thalidomide analogs: potent inhibitors of TNF- $\alpha$ production. Bioorg Med Chem Lett, 9:1625-30.

Raje N, Kumar S, Hideshima T, et al. 2004. Combination of the mTOR inhibitor Rapamycin and Revlimid (CC-5013) has synergistic activity in multiple myeloma. Blood, 104:4188-93.
Rajkumar SV, Jacobus S, Callander N, et al. 2006b. A randomized phase III trial of lenalidomide plus high-dose dexamethasone versus lenalidomide plus low-dose dexamethasone in newly diagnosed multiple myeloma (E4A03): a trial coordinated by the Eastern Cooperative Oncology Group. Blood, 108:239a.

Rajkumar SV, Blood E, Vesole D, et al. 2006a. Phase III clinical trial of thalidomide plus dexamethasone compared with dexamethasone alone in newly diagnosed multiple myeloma: a clinical trial coordinated by the Eastern Cooperative Oncology Group. J Clin Oncol, 24:431-6.

Rajkumar SV, Hayman SR, Lacy MQ, et al. 2005. Combination therapy with lenalidomide plus dexamethasone (Rev/Dex) for newly diagnosed myeloma. Blood, 106:4050-3.

Richardson P, Jagannath S, Schlossman R, et al. 2002. A multi-center, randomized, phase II study to evaluate the efficacy and safety of two CC-5013 dose regimens when used alone or in combination with dexameyhasone (Dex) for the treatment of relapsed or refractory multiple myeloma (MM). Blood, 100:104a.

Richardson PG, Blood E, Mitsiades CS, et al. 2006. A randomized phase 2 study of lenalidomide therapy for patients with relapsed or relapsed and refractory multiple myeloma. Blood, 108:3458-64.

Richardson PG, Schlossman RL, Weller E, et al. 2002. Immunomodulatory drug CC-5013 overcomes drug resistance and is well tolerated in patients with relapsed multiple myeloma. Blood, 100:3063-7.

Rowland TL, McHugh SM, Deighton J, et al. 2001. Differential effect of thalidomide and dexamethasone on the transcription factor NF- $\mathrm{KB}$. Int Immunopharmacol, 1:49-61.

Shannon E, Aseffa A, Pankey G, et al. 2000. Thalidomide's ability to augment the synthesis of IL-2 in vitro in HIV-infected patients is associated with the percentage of CD4+ cells in their blood. Immunopharmacology, 46:175-9.

Singhal S, Mehta J, Desikan R, et al. 1999. Antitumor activity of thalidomide in refractory multiple myeloma. $N$ Engl J Med, 341:1565-71.

Stewart AK, Scanga SE, Zhu YX, et al. 2004. Inhibition of Wnt pathway signaling by thalidomide and revlimid: studies in a drosophila model system. Blood, 104:916a.

Tai YT, Li XF, Catley L, et al. 2005. Immunomodulatory drug lenalidomide (CC-5013, IMiD3) augments anti-CD40 SGN-40-induced cytotoxicity in human multiple myeloma: clinical implications. Cancer Res, 65:11712-20.

Terpos E, Dimopoulos MA, Sezer O. 2007. The effect of novel antimyeloma agents on bone metabolism of patients with multiple myeloma. Leukemia, 21:1875-84.

Tseng S, Pak G, Washenik K, et al. 1996. Rediscovering thalidomide: a review of its mechanism of action, side effects, and potential uses. $J$ Am Acad Dermatol, 35:969-79.

Weber D, Wang M, Chen C, et al. 2006. Lenalidomide plus high-dose dexamethasone provides improved overall survival compared to highdose dexamethasone alone for relapsed or refractory multiple myeloma (MM): results of 2 phase III studies (MM-009, MM-010) and subgroup analysis of patients with impaired renal function. Blood, 108:1012a.

Wu A, Scheffler MR. 2004. Multi-dose phamacokinetics and safety of CC-5013 in 15 multiple myeloma patients. J Clin Oncol, 22:141s. 